\title{
Research Article \\ PID Controller of Sprayer Chassis by Sliding Mode
}

\author{
Hua Zhang \\ School of Mechanical Engineering, Institute of Intelligent Machinery and Agricultural Equipment, \\ Anhui Science and Technology University, Fengyang, China \\ Correspondence should be addressed to Hua Zhang; chinafeihong@163.com
}

Received 16 June 2017; Revised 27 September 2017; Accepted 17 October 2017; Published 9 November 2017

Academic Editor: Petko Petkov

Copyright (C) 2017 Hua Zhang. This is an open access article distributed under the Creative Commons Attribution License, which permits unrestricted use, distribution, and reproduction in any medium, provided the original work is properly cited.

In order to solve the straight line drive coordinated control problem of the four-wheel independent drive sprayer chassis, the dynamic model of sprayer chassis and electromagnetic proportional valve controlled hydraulic motor model are established. The additional yaw moment is designed to rectify the deviation with sliding mode variable structure control. PID control strategy is used to calculate the control voltage adjustment of the electromagnetic proportional valve. The simulation results show that the accumulative deviation of the chassis is $0.2 \mathrm{~m}$ out of $100 \mathrm{~m}$ when the coordinated control strategy is adopted on different adhesive coefficient pavement, which is much smaller than the value without control. The test results of test prototype show that the yaw acceleration of the chassis can be as low as $-0.0132 \mathrm{~m} / \mathrm{s}^{2}$ on different adhesive coefficient pavement with coordinated control, which is smaller than the value without control, and the straight line drive requirements are met. It is feasible to combine sliding mode variable structure with PID control and use the electromagnetic proportional control technology in the straight line drive coordinated control of sprayer chassis by adding the yaw moment to rectify the deviation of chassis based on the yaw acceleration detection.

\section{Introduction}

The spray operation of plant protection requires that the self-propelled sprayer chassis has good flexibility and crosscountry performance in modern complex agricultural and forestry environment [1-4]. To this end, the author proposes a fully hydraulic driven sprayer chassis [5], where four hydraulic motors are adopted to direct drive wheel walking and hydraulic cylinders are adopted to realize the adjustment of wheelbase, ground clearance, and the promotion and demotion of spray system. But the terrain is complex in field operation and the change of adhesive coefficient or fluctuation of pavement may cause the hydraulic independent drive chassis to deviate the straight line drive and the straight line drive stability is decreased. Therefore, it is necessary to develop a coordinated control strategy for four-wheel independent drive sprayer chassis to solve the straight line drive problem. Current researches mostly focus on coordinated control of four-wheel drive electric vehicle. For example, Moure and Kersting [6] develop a four-wheel torque distribution control method for eliminating motor fault and related driving factors' influence on the dynamic stability of the wheel independent electric vehicle and ensure the stability and safety of the car in the straight line driving process. Salama and Vantsevich [7] present a four-wheel speed control method based on longitudinal inverse dynamics of full electric wheel drive vehicle. The torque distribution of each wheel is coordinated and straight line driving stability and energy efficiency of the vehicle under random road are improved. Bode et al. [8] present a traction controller for electric wheel drive vehicle. The wheel longitudinal slip is kept in the best range and the vehicle straight line driving stability is ensured by adjusting the voltage of the motor and estimating the pavement adhesion force of each wheel. Other scholars have applied the additional yaw moment control and the quadratic optimal control to the deviation rectification control and stability control of the four-wheel drive electric vehicle, respectively, and have received good control results $[9,10]$. A 2-DOF vehicle dynamics model, an upper level controller, and a torque distribution algorithm are designed for the straight running stability control system for a fourwheel independent in-wheel-motor drive electric vehicle [11]. 
Considering the motor errors or fault tolerance, the PID control method based on BP neural net work was presented to coordinate torque of the wheels to improve the electric vehicles straight line running stability [12]. Some motor fault tolerance control strategies are proposed based on the performance of the distributed driving electric vehicles [13]. A control algorithm combining the sliding mode variable structure and optimization control method is proposed for straight line stability control of four-wheel drive vehicle, where the slip rates controller and yaw moment adjustment are adopted [14]. There are also some researches focusing on the deviation rectification control of vehicles. For example, traversal control [15] of intelligent vehicle is realized by tracking the desired yaw rate. Static liquid drive and steer of high speed tracked vehicle are realized by considering a variety safety conditions on ice road [16], which provide reference for the rectification of intelligent navigation vehicle and steering correction of hydraulic vehicles. The deviation of UAV is rectified by combining the main wheel differential brake and rudder skate $[17,18]$ and using the feedback principle, which also has reference for deviation rectification control of autonomous chassis control system. There are also other researches focusing on the deviation rectification of strip steel production line. For example, the intelligent control strategies such as fuzzy control and fuzzy PID control are used in the deviation rectification control of composite winding $[19,20]$ and electric hydraulic servo deviation control [21] is used in strip steel production line, which shows advantages of intelligent control algorithm and electrohydraulic servo control in deviation rectification system. In summary, in the above researches, the overall disturbances of the controlled object are not as good as that of the sprayer chassis and the straight line coordinated control of fully hydraulic driven sprayer chassis is more difficult. Firstly, the working condition of sprayer is more complex and the outer disturbance such as the different pavement adhesive coefficients and fluctuations of pavement is unavoidable. Secondly, the characteristics of electric motor and hydraulic motor are different. To some extent, the electric motor is more easily controlled than hydraulic motor because of its simple structure and nice control characteristics. Thirdly, many deviation rectification control strategies have poor real-time performance because of the inertia of control component and the computing time of complex algorithms [22]. Lastly, some control component such as servo valve in servo control system is expensive despite its high accuracy and rapid response. Therefore, considering the working conditions of sprayer chassis, the characteristics of hydraulic motor, the cost performance, and the rapid response of control system, the author provides the straight line coordinated control strategy to meet the straight line driving demand of four-wheel independent drive sprayer chassis. This strategy combines sliding mode variable structure control and PID control because of their simple algorithms and easier implementation. Furthermore, this strategy is based on yaw acceleration detection and electromagnetic proportional control technology to realize the additional yaw moment compensation because of its good cost performance and adaptability for hydraulic control system.

\section{Straight Line Drive Coordinated Control Strategy of Sprayer Chassis}

Straight line drive coordinated control is referring to the use of control strategy and control method, ensuring the coordinated operation of multiple wheel motor in different driving conditions, rectifying the deviation of the chassis real time, to further develop the hydraulic motor independent drive and control advantages, and making hydraulic wheel drive vehicles have excellent driving performance. Straight line drive coordinated control strategy of four-wheel hydraulic motor independent drive sprayer chassis is formulated [23] (shown in Figure 1), based on which the dynamic model of the sprayer chassis is established. The yaw rate of the chassis and the slip ratio of each wheel are obtained. The straight line driving state of the chassis is judged according to the yaw rate. The additional yaw moment to rectify the yaw motion is calculated if the yaw rate exceeds the preset threshold; meanwhile, the slip ratio of front and rear wheel at the same side is identical. PID control strategy is used for converting to control voltage adjustment of electromagnetic proportional valve. Taking the slip ratio deviation being 0 as the control target, PID control is carried out on front and rear wheel at the same side if the wheel slip ratio deviation of front and rear wheel at the same side exceeds the preset threshold. Thus the control voltage adjustment of electromagnetic proportional valve corresponding to hydraulic motor is calculated. Antislip-regulation with PID is carried out separately if the wheel is excessively slipping and the control voltage adjustment of the electromagnetic proportional valve is calculated. Thereby, the driving moment balance of both sides of the wheel is dynamically adjusted and the goal that the chassis keeps driving in straight line is achieved.

\section{Straight Line Drive Coordinated Control System Modeling of Sprayer Chassis}

3.1. Dynamic Model Establishment of Sprayer Chassis. The influence of wind resistance can be neglected because of the low speed of spray operation. The dynamic model of sprayer chassis is established synthesizing the road model, tire model, suspension model, wheel hydraulic motor model, and hydraulic chassis model as follows:

$$
\begin{aligned}
& M\left(\dot{V}_{x}-V_{y} \omega_{r}+V_{Z} \dot{\theta}\right)=F_{x 1}+F_{x 2}+F_{x 3}+F_{x 4} \\
& M\left(\dot{V}_{y}+V_{x} \omega_{r}-V_{Z} \dot{\varphi}\right)=F_{y 1}+F_{y 2}+F_{y 3}+F_{y 4} \\
& M_{b}\left(\dot{V}_{z}-V_{x} \dot{\theta}+V_{y} \dot{\varphi}\right)=F_{s 1}+F_{s 2}+F_{s 3}+F_{s 4}-M_{b} g \\
& I_{Z} \dot{\omega}_{r} \\
& =\frac{B}{2\left(F_{x 2}+F_{x 4}-F_{x 3}-F_{x 1}\right)}+l_{f}\left(F_{y 1}+F_{y 2}\right) \\
& \quad-l_{r}\left(F_{y 3}+F_{y 4}\right)
\end{aligned}
$$




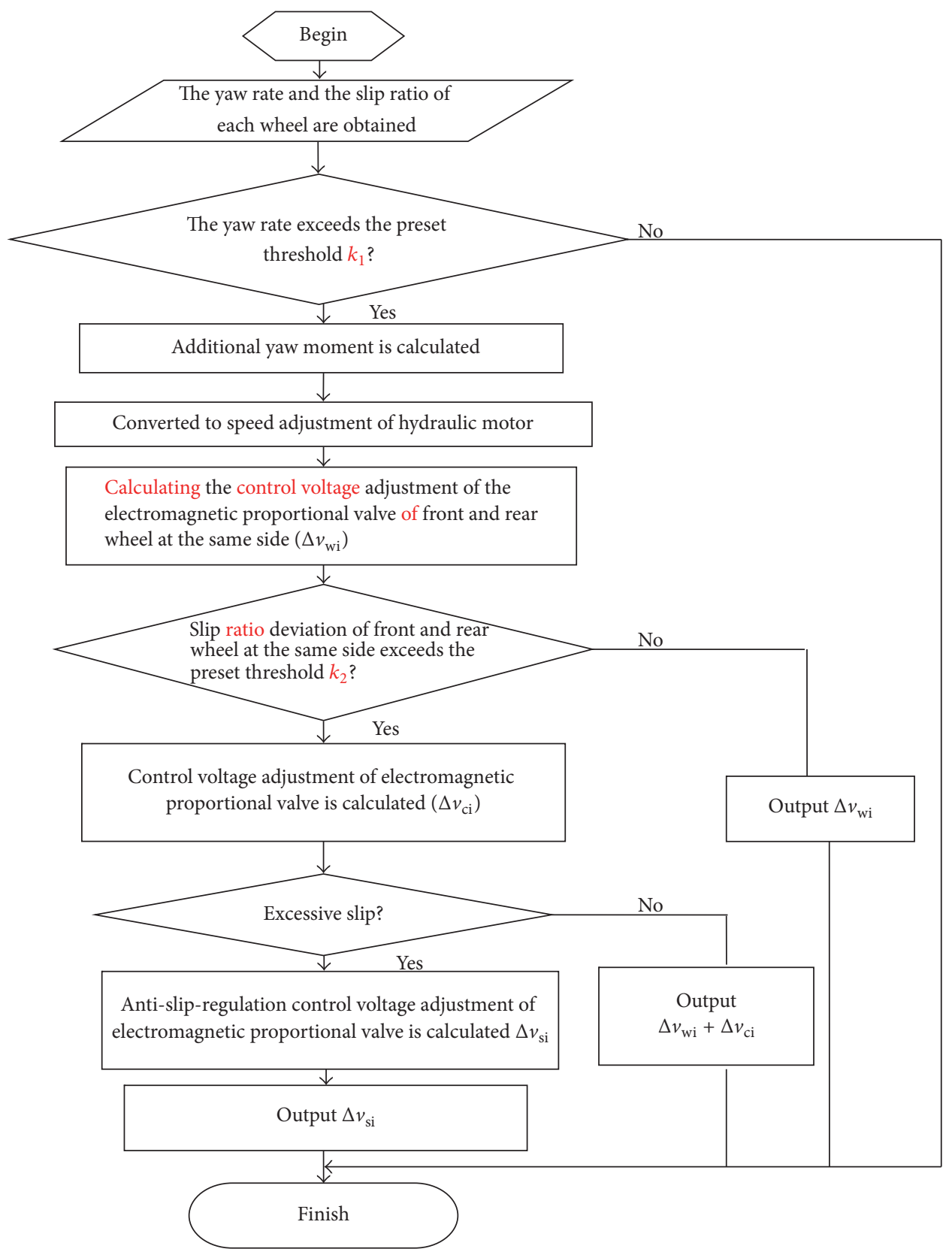

FIGURE 1: Straight line drive coordinated control strategy of sprayer chassis.

$$
\begin{aligned}
& I_{x} \ddot{\varphi} \\
& F_{\mathrm{si}}=k_{\mathrm{si}}\left(z_{\mathrm{ti}}-z_{i}\right)+b_{\mathrm{si}}\left(\dot{z}_{\mathrm{ti}}-\dot{z}_{i}\right) \\
& =\frac{B}{2\left(F_{s 1}+F_{s 3}-F_{s 2}-F_{s 4}\right)} \\
& +h_{x}\left(F_{y 1}+F_{y 2}+F_{y 3}+F_{y 4}\right) \\
& F_{\mathrm{xi}} \\
& =\left[1-\exp \left(-\phi_{i}-E \phi_{i}^{2}-\left(E^{2}+\frac{1}{12}\right) \phi_{i}^{3}\right)\right] \frac{\phi_{\mathrm{xi}}}{\phi_{i}} \mu_{\mathrm{xi}} F_{\mathrm{zi}} \\
& I_{y} \ddot{\theta} \\
& =l_{r}\left(F_{s 3}+F_{s 4}\right)-l_{f}\left(F_{s 1}+F_{s 2}\right) \\
& -h_{y}\left(F_{x 1}+F_{x 2}+F_{x 3}+F_{x 4}\right) \\
& F_{\mathrm{yi}} \\
& =\left[1-\exp \left(-\phi_{i}-E \phi_{i}^{2}-\left(E^{2}+\frac{1}{12}\right) \phi_{i}^{3}\right)\right] \frac{\phi_{\mathrm{yi}}}{\phi_{i}} \mu_{\mathrm{yi}} F_{\mathrm{zi}} \\
& X=\int V_{x} d t
\end{aligned}
$$




$$
\begin{aligned}
& Y=\int V_{y} d t \\
& \phi_{\mathrm{xi}}=\frac{K_{\mathrm{xi}} S_{\mathrm{xi}}}{\mu_{\mathrm{xi}} F_{\mathrm{zi}}} \\
& \phi_{\mathrm{yi}}=\frac{K_{\mathrm{yi}} S_{\mathrm{yi}}}{\mu_{\mathrm{yi}} F_{\mathrm{zi}}} \\
& \phi_{i}=\sqrt{\phi_{\mathrm{xi}}{ }^{2}+\phi_{\mathrm{yi}}{ }^{2}},
\end{aligned}
$$

where $M$ is chassis mass. $F_{\mathrm{xi}}$ is wheel drive force. $V_{x}$ is chassis longitudinal velocity. $V_{y}$ is chassis lateral velocity. $\omega_{r}$ is chassis yaw rate. $\theta$ is chassis pitch angle. $\varphi$ is chassis heeling angle. $F_{\mathrm{yi}}$ is wheel lateral force. $M_{b}$ is chassis spring mass. $V_{z}$ is chassis vertical velocity. $l_{f}$ and $l_{r}$ are the distance from front and rear axle to the centroid, respectively. $B$ is wheel distance. $F_{\text {si }}$ is suspension force. $h_{x}$ and $h_{y}$ are the distance from the centroid to the roll axis and the pitch axis, respectively. $I_{x}$, $I_{y}$, and $I_{z}$ are chassis roll, pitch, and yaw moment of inertia, respectively. $z_{\mathrm{ti}}$ is wheel centroid height. $z_{i}$ is centroid height of chassis spring mass. $k_{\mathrm{si}}$ is chassis suspension stiffness. $b_{\mathrm{si}}$ is chassis suspension damping. $E$ is total tangential force curvature factor. $\phi_{\mathrm{xi}}, \phi_{\mathrm{yi}}$, and $\phi_{i}$ are longitudinal slip ratio of wheel, lateral slip ratio, and dimensionless total slip ratio, respectively. $K_{\mathrm{xi}}$ and $K_{\mathrm{yi}}$ are longitudinal slipping stiffness and cornering stiffness, respectively. $\mu_{\mathrm{xi}}$ and $\mu_{\mathrm{yi}}$ are longitudinal and transverse friction coefficient of tire, respectively. $F_{\mathrm{zi}}$ is vertical force of wheel. $X$ is straight line displacement; $Y$ is lateral deviation $(i=1,2,3$, and 4 are the left front wheel, the right front wheel, the left rear wheel, and the right rear wheel of the chassis, resp.).

3.2. Model of Electromagnetic Proportional Valve Controlled Hydraulic Motor. According to the flow equation of electromagnetic proportional valve, motor cavity flow continuity equation, torque balance equation of motor, the input and output model of electromagnetic proportional valve, and load torque equation, the model of electromagnetic proportional valve controlled hydraulic motor is established as follows:

$$
\begin{aligned}
Q_{L} & =K_{q} x_{v}-K_{c} P_{L} \\
Q_{L} & =D_{m} \frac{d \theta_{m}}{d t}+C_{\mathrm{tm}} P_{L}+\frac{V_{t}}{4 \beta_{e}} \frac{d P_{L}}{d t} \\
D_{m} P_{L} & =J_{t} \frac{d^{2} \theta_{m}}{d t^{2}}+B_{m} \frac{d \theta_{m}}{d t}+G \theta_{m}+T_{L} \\
x_{v} & =K_{e} K_{\mathrm{pv}} v_{i} \\
T_{L} & =F_{\mathrm{xi}} R_{\mathrm{ei}}+T_{f i} \\
R_{\mathrm{ei}} & =\frac{\sin \left\{\cos ^{-1}\left(\Delta R / R_{C}\right)\right\}}{\cos ^{-1}\left(\Delta R / R_{C}\right)} \bullet R_{C} \\
T_{\mathrm{fi}} & =W_{i} \bullet 0.1 \alpha_{i}
\end{aligned}
$$

$$
\alpha_{i}=\tan ^{-1}\left(\frac{V_{\mathrm{yi}}}{V_{\mathrm{xi}}}\right) \text {, }
$$

where $Q_{L}$ is load flow. $P_{L}$ is load pressure difference. $K_{q}$ is flow gain of proportional valve. $K_{c}$ is flow pressure coefficient of proportional valve. $x_{v}$ is spool displacement. $D_{m}$ is volumetric displacement of motor. $\theta_{m}$ is angle position of motor shaft. $C_{\mathrm{tm}}$ is total leakage coefficient of motor. $V_{t}$ is the total volume of two cavities. $\beta_{e}$ is system effective volume modulus. $J_{t}$ is total inertia of motor and load. $B_{m}$ is load coefficient of viscous damping. $G$ is torsion spring gradient. $T_{L}$ is load torque. $K_{e}$ is amplifier gain. $K_{\mathrm{pv}}$ is flow gain of electromagnetic proportional valve. $v_{i}$ is control voltage. $R_{\mathrm{ei}}$ is rolling radius. $T_{\mathrm{fi}}$ is rolling resistance torque. $\alpha_{i}$ is distortion angle. $R_{c}$ is wheel radius. $W_{i}$ is the vertical force on the wheels exerted by the ground.

3.3. Straight Line Drive Coordinated Control Model of Sprayer Chassis. The sliding mode variable structure control method [24] is adopted and the switching surface function is designed based on the yaw angle, yaw rate, and yaw angle acceleration of the chassis as follows:

$$
S(x)=\omega_{r}+k_{1} \dot{\omega}_{r}+k_{2} \int_{0}^{t} \omega_{r} d t,
$$

where $k_{1}$ and $k_{2}$ are weight coefficients.

Additional yaw moment for rectifying the deviation is designed as follows:

$$
\begin{aligned}
\Delta M_{Z}= & l_{f} F_{\mathrm{yf}}-l_{r} F_{\mathrm{yr}} \\
& -I_{Z}\left(k_{1} \ddot{\omega}_{r}+k_{2} \omega_{r}+k_{3} S+\varepsilon|S| \operatorname{sgn}(S)\right),
\end{aligned}
$$

where $k_{3}$ and $\varepsilon$ are adjustment parameters of sliding mode variable structure controller.

The additional yaw moment is assigned to the left and right wheels according to the negative and positive opposite mode. The relationship between the wheel drive force adjustment and the additional yaw moment can be expressed as follows:

$$
\Delta F_{\mathrm{xi}}=(-1)^{i} \frac{\Delta M_{Z}}{2 B} .
$$

The relationship between the torque adjustment of the hydraulic motor and the adjustment of the driving force of the wheel can be expressed as follows:

$$
\Delta T_{\mathrm{ei}}=R_{\mathrm{ei}} \Delta F_{\mathrm{xi}} .
$$

The relationship between the output torque adjustment of the hydraulic motor and the control voltage adjustment of the electromagnetic proportional valve is approximately linear. The relationship can be expressed as follows:

$$
\Delta T_{\mathrm{ei}}=-K_{T} \Delta V_{\mathrm{wi}} .
$$

Thus, control voltage adjustment of electromagnetic proportional valve in response to additional yaw moment can be expressed as follows:

$$
\Delta V_{\mathrm{wi}}=(-1)^{i-1} \frac{R_{\mathrm{ei}}}{2 B K_{T}} \Delta M_{Z},
$$


where $\Delta V_{\text {wi }}$ is control voltage adjustment of electromagnetic proportional valve. $K_{T}$ is torque coefficient. $\Delta M_{Z}$ is additional yaw moment.

When the slip ratio of the front and rear wheel of the same side is over the threshold, the voltage adjustments of the four electromagnetic proportional control valves can be expressed as follows:

$$
\begin{aligned}
e_{1} & =S_{x 3}-S_{x 1} \\
e_{2} & =S_{x 4}-S_{x 2} \\
\Delta V_{c 1} & =k_{\mathrm{cp}} e_{1}+k_{\mathrm{ci}} \int e_{1} d t+k_{\mathrm{cd}} \frac{d e_{1}}{d t} \\
\Delta V_{c 2} & =k_{\mathrm{cp}} e_{2}+k_{\mathrm{ci}} \int e_{2} d t+k_{\mathrm{cd}} \frac{d e_{2}}{d t} \\
\Delta V_{c 3} & =-\Delta V_{c 1} \\
\Delta V_{c 4} & =-\Delta V_{c 2} \\
S_{\mathrm{xi}} & =\frac{v_{\mathrm{ci}}-V_{\mathrm{xi}}}{v_{\mathrm{ci}}} \times 100 \%,
\end{aligned}
$$

where $S_{\mathrm{xi}}$ is wheel slip ratio. $v_{\mathrm{ci}}$ is rolling velocity of wheel center.

Total voltage adjustment can be expressed as follows:

$$
\Delta V_{i}=\Delta V_{\mathrm{wi}}+\Delta V_{\mathrm{ci}}
$$

The control voltage adjustment of proportional valve when the wheel is excessively slipping can be expressed as follows:

$$
\begin{aligned}
\Delta V_{\mathrm{si}} & =k_{\mathrm{SP}} e_{\mathrm{si}}+k_{\mathrm{si}} \int e_{\mathrm{si}} d t+k_{\mathrm{sd}} \frac{d e_{\mathrm{si}}}{d t} \\
e_{\mathrm{si}} & =S_{p}-S_{\mathrm{xi}},
\end{aligned}
$$

where $e_{1}$ and $e_{2}$ are slip ratio deviation of front and rear wheel at left and right side, respectively. $k_{\mathrm{cp}}, k_{\mathrm{ci}}$, and $k_{\mathrm{cd}}$ are parameters of PID controller. $e_{\mathrm{si}}$ is slip ratio deviation of wheel. $S_{P}$ is slip ratio threshold of wheel. $k_{\mathrm{sp}}, k_{\mathrm{si}}$, and $k_{\mathrm{sd}}$ are parameters of PID controller for anti-slip-control of single wheel.

\section{Straight Line Drive Coordinated Control Algorithm of Sprayer Chassis and System Simulation}

4.1. Straight Line Drive Coordinated Control Algorithm of Sprayer Chassis. The straight line drive coordinated control algorithm of sprayer chassis is as follows:

(1) Suspension force $F_{\mathrm{si}}$ and wheel vertical load $F_{\mathrm{zi}}$ are calculated by inputting centroid height of chassis spring mass $z_{i}$ and wheel centroid height $z_{\mathrm{ti}}$.

(2) We calculate the slip ration $S_{\mathrm{xi}}$ by wheel angular $\omega_{\mathrm{ci}}$ and the tire fore $F_{\mathrm{xi}}, F_{\mathrm{yi}}$ by inputting pavement adhesive coefficient $u_{i}$, heeling angle $\varphi_{i}$ and wheel vertical load $F_{\mathrm{zi}}$.

(3) The chassis' longitudinal speed $V_{x}$, lateral acceleration $V_{y}$, yaw rate $\omega_{r}$, and other state parameters are calculated by inputting the tire force $F_{\mathrm{xi}}, F_{\mathrm{yi}}$ and suspension force $F_{\mathrm{si}}$.
TABLE 1: Simulation parameters of straight line drive coordinated control of sprayer chassis.

\begin{tabular}{lc}
\hline Parameters & Value \\
\hline Chassis mass $M / \mathrm{kg}$ & 540 \\
Chassis spring mass $M_{b} / \mathrm{kg}$ & 460 \\
Roll moment of inertia $I_{x} /\left(\mathrm{kg} \cdot \mathrm{m}^{2}\right)$ & 350 \\
Pitching moment of inertia $I_{y} /\left(\mathrm{kg} \cdot \mathrm{m}^{2}\right)$ & 730 \\
Yaw moment of inertia $I_{z} /\left(\mathrm{kg} \cdot \mathrm{m}^{2}\right)$ & 640 \\
Damping coefficient of front suspension $b_{\mathrm{sf}}$ & 1000 \\
Damping coefficient of rear suspension $b_{\mathrm{sr}}$ & 1000 \\
Wheel centroid height $z_{\mathrm{ti}} / \mathrm{m}$ & 0.3 \\
Distance from front axle to the centroid $L_{f} / \mathrm{m}$ & 0.75 \\
Distance from rear axle to the centroid $L_{r} / \mathrm{m}$ & 0.75 \\
Wheel moment of inertia $J /\left(\mathrm{kg} \cdot \mathrm{m}^{2}\right)$ & 1.8 \\
Wheel distance $B / \mathrm{m}$ & 1.2 \\
Front suspension spring stiffness $k_{\mathrm{sf}} /\left(\mathrm{N} \cdot \mathrm{m}^{-1}\right)$ & 12000 \\
Rear suspension spring stiffness $k_{\mathrm{sr}} /\left(\mathrm{N} \cdot \mathrm{m}^{-1}\right)$ & 12000 \\
Longitudinal and transverse friction coefficient of tire $\mu_{x i}$ & $0.1,0.9$ \\
Centroid height of chassis spring mass $z_{i} / \mathrm{m}$ & 1.2 \\
\hline
\end{tabular}

(4) The control voltage adjustment of proportional valve $V_{i}$ is calculated by inputting chassis yaw rate $\omega_{r}$, wheel slip ratio $S_{\mathrm{xi}}$, front and rear axle wheel side force $F_{\mathrm{yf}}, F_{\mathrm{yr}}$, and other information in straight line drive coordinated control module.

If $\omega_{r} \leq k_{1}\left(k_{1}=0.01\right)$, the chassis can be kept driving in straight line and coordinated control does not work; else if $\omega_{r}>k_{1}$ and $\left|e_{x}\right| \leqslant k_{2}\left(k_{2}= \pm 0.0001\right), \Delta v_{\text {wi }}$ is output; else if $\omega_{r}>k_{1},\left|e_{x}\right|>k_{2}$, and $S_{\mathrm{xi}} \leqslant S_{p}\left(S_{p}=0.2\right), \Delta v_{\mathrm{wi}}+\Delta v_{\mathrm{ci}}$ is output; else if $\omega_{r}>k_{1},\left|e_{x}\right|>k_{2}$, and $S_{\mathrm{xi}}>S_{p}, \Delta v_{\mathrm{si}}$ is output.

(5) By inputting voltage control signal $V_{i}$, wheel rolling resistance moment $T_{\mathrm{fi}}$, rolling radius $R_{\mathrm{ei}}$, and driving force $F_{\text {xi }}$, four-wheel rotation angular speed $\dot{\theta_{m}}$ is calculated and output in valve controlled motor module.

4.2. System Simulation. According to the coordinated control algorithm above, the simulation model of straight line drive coordinated control of sprayer chassis is established in SIMULINK (shown in Figure 2).

4.3. Simulation Parameters Setting. The simulation parameters are set according to physical characteristics of the sprayer chassis test prototype and the test pavement (shown in Table 1).

4.4. Simulation Results and Analysis. The pavement adhesive coefficients of the wheel at the left and right side of the sprayer chassis are set as 0.1 and 0.9 , respectively [25]. The deviation of the sprayer chassis is measured by 100 meters' straight line displacement.

It can be seen that the left wheel is excessive slipping at the starting instant because of lower wheel speed, larger driving moment, and lower adhesive coefficient of pavement. The driving force of the left and right wheels is unbalanced and a yaw motion of the chassis is produced. The chassis 


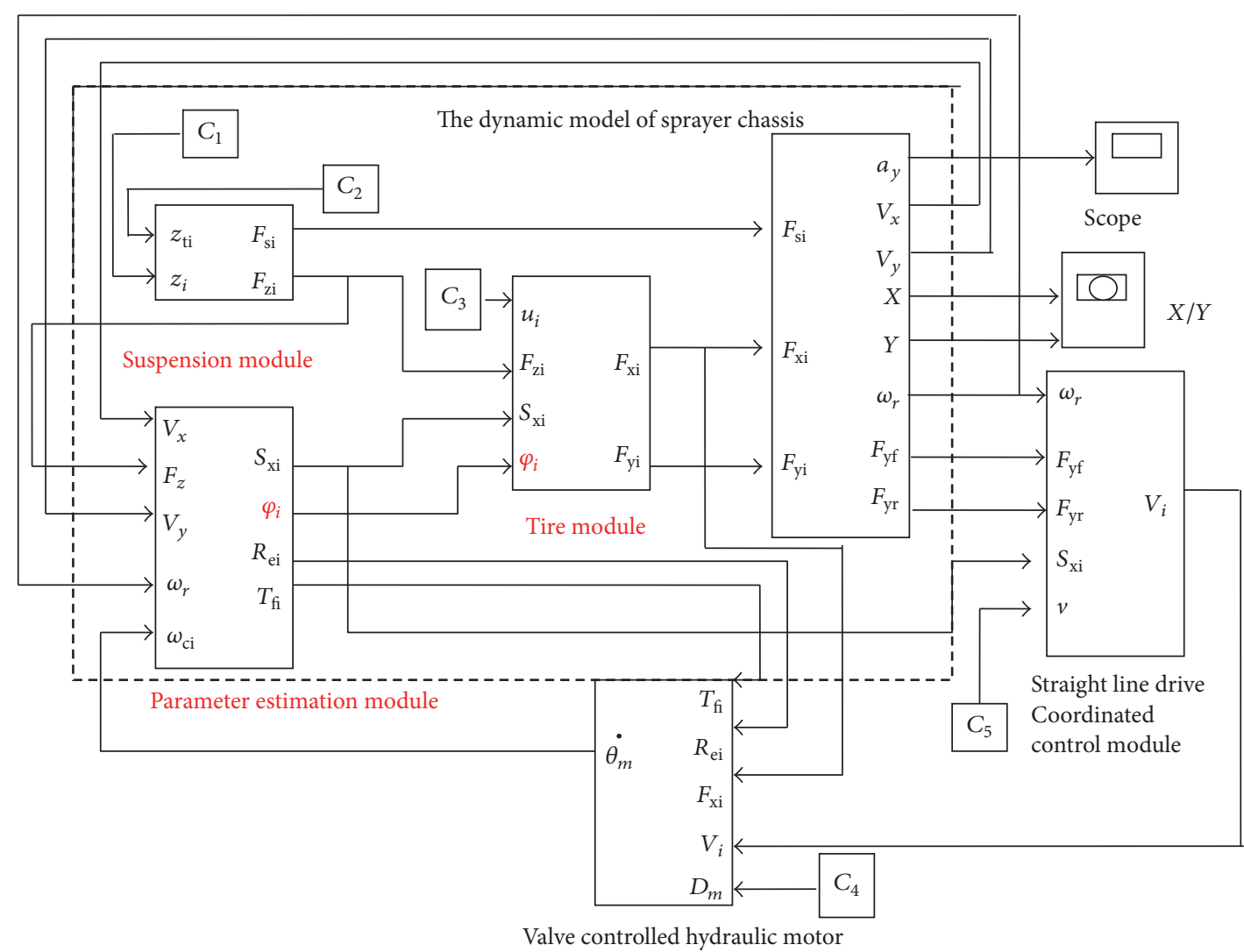

FIGURE 2: Simulation model of straight line drive coordinated control of sprayer chassis.

deviates from the straight line trajectory and will deviate continuously if without control. The cumulative deviation in 100 meters' straight line displacement is as much as $10 \mathrm{~m}$ (shown in Figure 3). But, under traditional PID control, the deviation is restrained and stabilized in the vicinity of 1 meter. Obviously, the chassis deviates from the straight line trajectory but will not deviate continuously. Slight deviation of chassis is also produced if straight line drive coordinated control strategy is adopted, but the real-time additional yaw moment compensation controlled by the proportional valve always works and the maximum deviation is no more than $0.2 \mathrm{~m}$, which shows that the coordinated control strategy has better straight line drive control effect on different adhesive coefficient pavement.

\section{Experiment Research}

The four-wheel independent drive sprayer chassis test prototype is made and the acceleration test system is installed (shown in Figure 4). The hydraulic workstation provides hydraulic drive force for the entire chassis hydraulic system. The wheels are driven directly by 4 -wheel motors. The clearance is adjusted by clearance adjustment hydraulic cylinders and the wheelbase is adjusted by wheelbase adjustment hydraulic cylinders. The yaw acceleration of the chassis is detected by yaw acceleration sensor.

Two different adhesive coefficient pavements are laid underneath the wheels in order to verify the effect of the

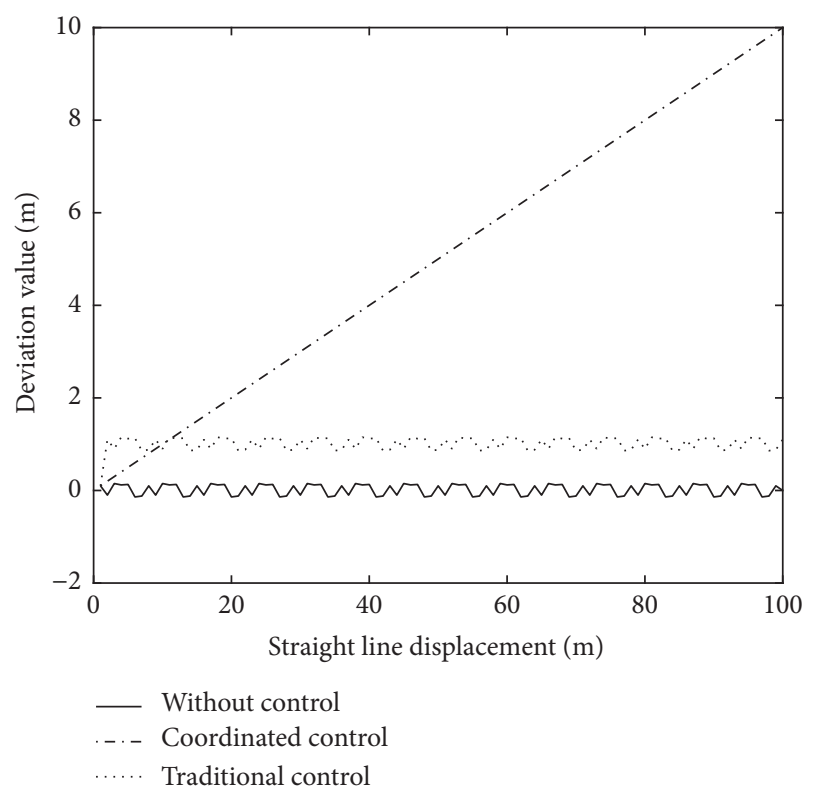

FIGURE 3: Simulation results of the straight line drive coordinated control on different adhesive coefficients pavement.

straight line drive coordinated control of the chassis. Lower adhesive coefficient pavement is simulated with plexiglas at the left side and higher adhesive coefficient pavement is 


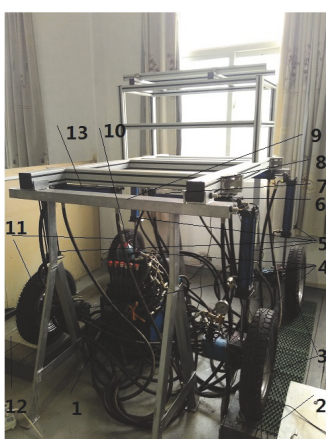

(a) Test prototype of sprayer chassis

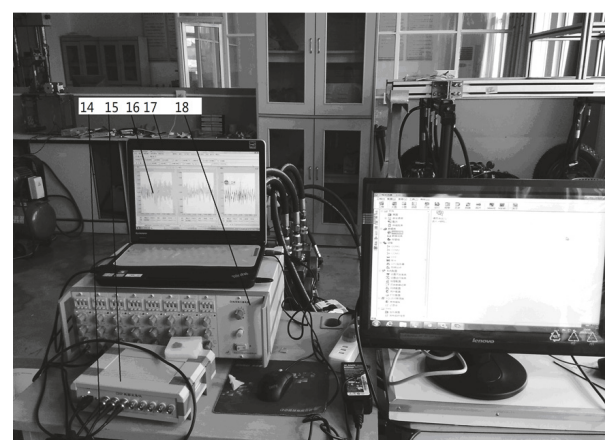

(b) Acceleration test system

Figure 4: Test prototype of four-wheel independent drive sprayer chassis. (1) Hydraulic workstation. (2) Rough pavement. (3) Right side wheel. (4) Wheel motor. (5) Clearance adjustment hydraulic cylinder. (6) Yaw acceleration sensor. (7) Electronic ruler. (8) Chassis frame. (9) Chassis bench. (10) Accumulator. (11) Left side wheel. (12) Smooth pavement. (13) Wheelbase adjustment hydraulic cylinder. (14) Connecting cable. (15) Data acquisition instrument. (16) Signal acquisition and analysis laboratory software system. (17) Computer. (18) Double integrating charge amplifier.

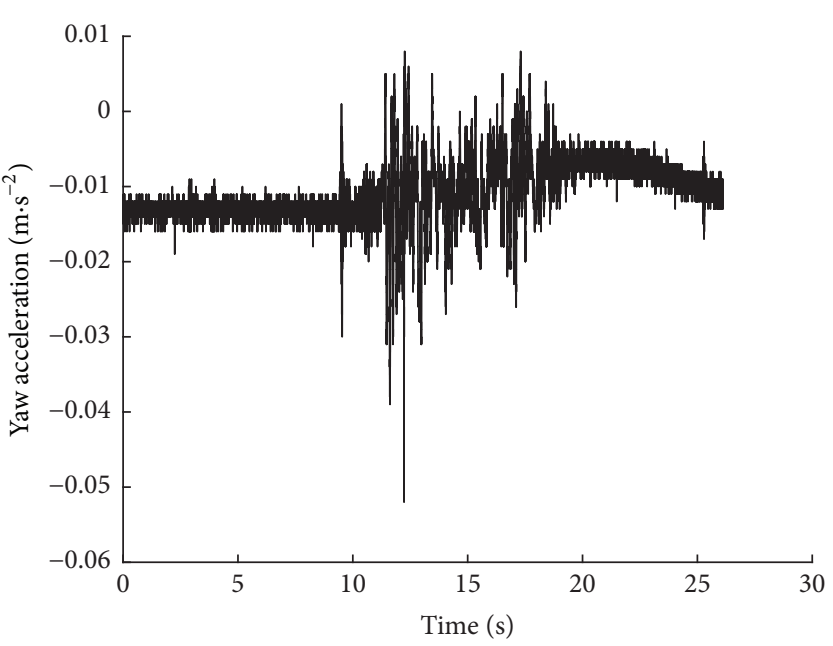

(a) Without control

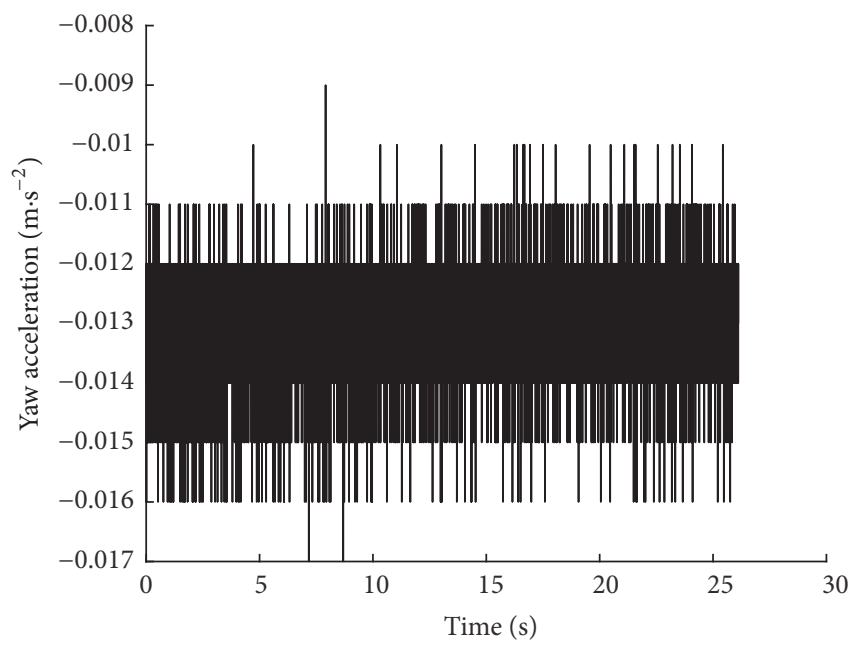

(b) Coordinated control

FIGURE 5: Measure results of yaw acceleration of chassis.

simulated with antiskid pad at the right side. The contact pressure between the tire and the pavement is adjusted equally through clearance adjustment hydraulic cylinders. The maximum driving torque on lower adhesive coefficient pavement is less than that on higher adhesive coefficient pavement due to slipping. Therefore, the driving torques on wheels at two sides are unbalanced, resulting in the yaw motion of the chassis. The change of the chassis yaw acceleration is measured in real time by yaw acceleration sensor.

At the time of $0-8 \mathrm{~s}$, chassis is on the same adhesive coefficient pavement. The yaw acceleration center value is in the vicinity of $-0.013 \mathrm{~m} / \mathrm{s}^{2}$ and straight line drive can be kept. The chassis drives on different adhesive coefficient pavement at $8 \mathrm{~s}$, whose yaw acceleration has obvious fluctuation and the peak value can reach $-0.05 \mathrm{~m} / \mathrm{s}^{2}$ if without control (shown in Figure 5(a)). The chassis will gradually deviate from the direction of the straight line. But when adopting chassis straight line drive coordinated control strategy, the additional yaw moment generating from hydraulic motor can effectively counteract the chassis yaw moment because of the real-time regulation of electromagnetic proportional valve. The yaw acceleration center value of the chassis is in the vicinity of $-0.0132 \mathrm{~m} / \mathrm{s}^{2}$ (shown in Figure 5(b)) and the chassis can be kept straight line driving. The rectifying deviation effect in straight line drive is good under coordinated control strategy.

\section{Conclusion}

In this paper, a hydraulic motor independent drive sprayer chassis is proposed and its straight line drive coordinated control strategy is studied in order to improve the straight line 
drive stability. Theoretical analysis and prototype test are used to demonstrate the effect of coordinated control of sprayer chassis. The conclusions are as follows.

(1) Based on the chassis yaw acceleration test, the electromagnetic proportional control technique is applied to compensate the yaw moment of the chassis by combining sliding mode variable structure control and PID control, which is beneficial to the real-time rectifying deviation of the chassis and has good cost performance. Furthermore, the sprayer chassis' straight line drive stability is improved under the coordinated control strategy. The simulation and test results confirm the effectiveness of the control strategy.

(2) The acceleration of the chassis is measured by the acceleration test system, which reflects the trend of the yaw motion of the chassis and its value can be used as the basis for judging the effect of the straight line drive coordinated control of the chassis.

(3) The mathematical computing ability of PLC used in this paper is rapid but less than DSP. So, DSP + PLC may be more appropriate $\mathrm{CPU}$ in order to shorten the response time further. The next work may be algorithm implementation with DSP and PLC. The response time detection and comparison need to be done thoroughly.

\section{Conflicts of Interest}

The author declares that there are no conflicts of interest regarding the publication of this paper.

\section{Acknowledgments}

This research is supported by the Natural Science Research Project of Anhui Higher Education Institutions (Grant no. KJ2017A508) and Doctoral Initial Funding of Anhui Science and Technology University.

\section{References}

[1] J. Matthew and A. Steve, "High clearance adjustable sprayer," US, US9737066B2, 2017.

[2] H. Y. Zhou, S. M. Liu X, X. J. Yang, and H. R. Yan, "Development on field vegetables high clearance self-propelled boom sprayer," Journal of Agricultural Mechanization Research, vol. 33, no. 06, pp. 70-72, 2011.

[3] D. Lu, Research and development for flexible chassis and its control system of conservative work machines, Northwest AF University, Shanxi, China, 2011.

[4] J. Z. Liu, "Self propelled high clearance adjustable hydraulic drive chassis of multifunctional variable sprayer for paddy," China, CN201821818U, 2011.

[5] H. Zhang, J. Q. Zheng, and Y. L. Xu, "Intelligent multifunctional sprayer chassis, China," ZL10290793.0[P], pp. 2014-04, 2013.

[6] C. Moure and K. Kersting, "Development of functional safety in a multi-motor control system for electric vehicles," $S A E$ Technical Papers, 2012.

[7] M. Salama and V. V. Vantsevich, "A parallel control of four independently driven wheels to maintain UGV inverse dynamics," in Proceedings of the ASME International Mechanical
Engineering Congress and Exposition, IMECE 2014, November 2014.

[8] K. Bode, K. Jalali, S. Lambert et al., "Design of an advanced traction controller for an electric vehicle equipped with four direct driven in-wheel motors," in Proceedings of the World Congress, 2008.

[9] X. C. Huang, Coordinated control for straight driving stability of four wheel independent drive electric vehicle, Jiangsu University, Jiangsu, China, 2015.

[10] H. W. Liu, The Stability Control Method of Four-Wheel Independent Drive Electric Vehicle on Different Adhesive Conditions, Yanshan University, Hebei, China, 2013.

[11] Y. Cao, L. Zhai, T. Sun, and H. Gu, "Straight Running Stability Control Based on Optimal Torque Distribution for a Four inwheel Motor Drive Electric Vehicle," Energy Procedia, vol. 105, pp. 2825-2830, 2017.

[12] H. H. Zhang, Q. N. Wang, and L. Q. Jin, "Study on the straightline running stability of the four-wheel independent driving electric vehicles," in Proceedings of the Asia Pacific Automotive Engineering Conference, 2007.

[13] X. Liu, H. He, R. Xiong, and Q. Tong, "Study on the fault tolerance control strategy of the distributed driving electric vehicle running in straight line with failure motor," Journal of Paediatrics \& Child Health, vol. 29, no. 5, pp. 344-349, 2012.

[14] Z. Wang, Y. Zhou, C. Li, and J. Fang, "Research on straight line stability control strategy of four wheel drive vehicle based on the sliding mode variable structure control and optimization algorithm," Energy Procedia, vol. 104, pp. 342-347, 2016.

[15] J. Wang, W. Chen, T. Wang, M. Wang, and L. Xiao, "Vision guided intelligent vehicle lateral control based on desired yaw rate," Jixie Gongcheng Xuebao/Journal of Mechanical Engineering, vol. 48, no. 4, pp. 108-115, 2012.

[16] L. Yang, B. Ma, and H.-Y. Li, "Steering safety control strategy of hydrostatic driving tracked vehicle on icy-snowy road," Journal of Jilin University, vol. 41, no. 4, pp. 904-909, 2011.

[17] Y. Wang and Y. X. Wang, "Lateral deviation correction control for UAV taxiing," Acta Aeronautica Et Astronautica Sinica, vol. 29, pp. S142-S149, 2008.

[18] Y.-P. Deng and Y.-M. Fan, "Design and simulation of ground taxiing system for UAV with four wheels," Journal of System Simulation, vol. 20, no. 21, pp. 5929-5939, 2008.

[19] X. He, Y. Shi, and X. Qin, "Research on technology of lateral position control in composites tape winding," Journal of Northwestern Polytechnical University, vol. 32, no. 3, pp. 464-469, 2014.

[20] K. Yang, Y. Shi, X. He, and L. Yan, "Controlling technology of intelligent position correcting in winding process of composites," Acta Aeronautica et Astronautica Sinica, vol. 32, no. 7, pp. 1318-1325, 2011.

[21] L. Quan, M. Li, L. Yao, and X. Xu, "Electro-hydraulic servo edge position control technology with low energy lose and key devices redundancy," Jixie Gongcheng Xuebao/Journal of Mechanical Engineering, vol. 45, no. 12, pp. 53-58, 2009.

[22] J. H. Jiang, R. Y. Zhu, and M. T. Yang, "Intelligent deviation rectifying control of the multi-row vertical-forming packaging machine," Packaging Engineering, vol. 25, no. 4, pp. 187-188, 2004.

[23] H. Zhang, Research on the hydraulic control system of flexible intelligent sprayer chassis, Nanjing Forestry University, Jiangsu, China, 2016. 
[24] Y. M. Fang, Z. J. Wang, Y. P. Xie, and X. H. Jiao, "Sliding mode variable structure control of multi-model switching for rolling mill hydraulic servo position system," Electric Machines and Control, vol. 14, no. 5, pp. 91-96, 2010.

[25] G. R. Zhuo, Key Technology Research of Four Wheel Drive Electric Vehicle, Tongji University, Shanghai, China, 2004. 


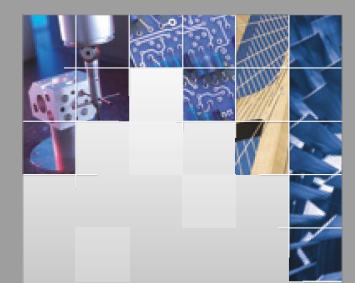

\section{Enfincering}
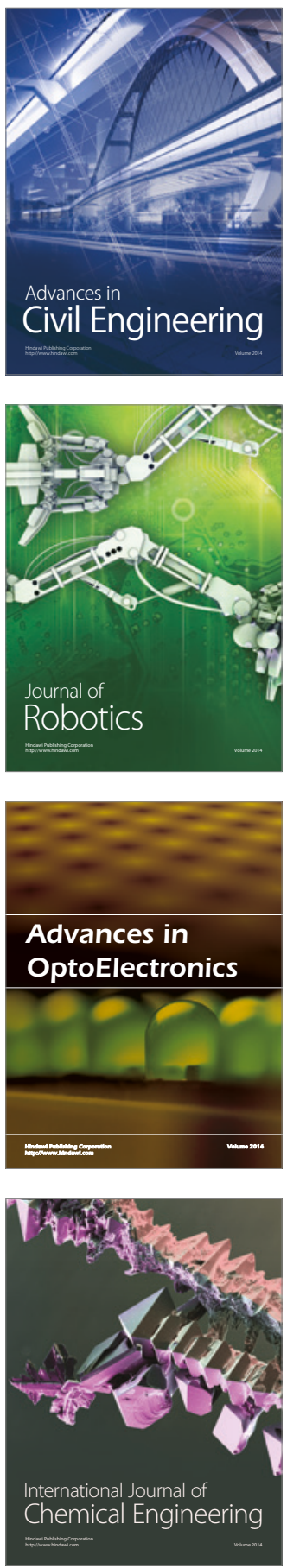

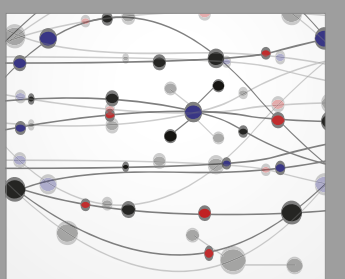

The Scientific World Journal

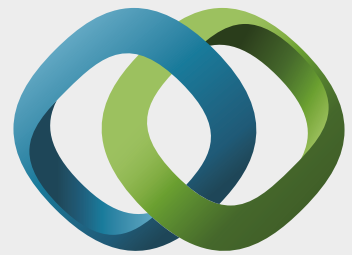

\section{Hindawi}

Submit your manuscripts at

https://www.hindawi.com
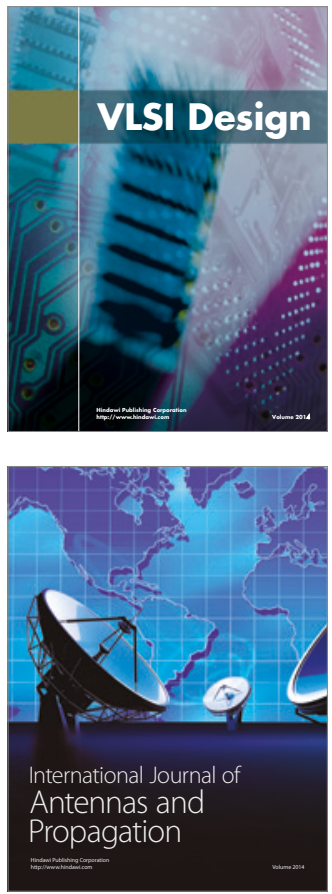

\section{Rotating}

Machinery
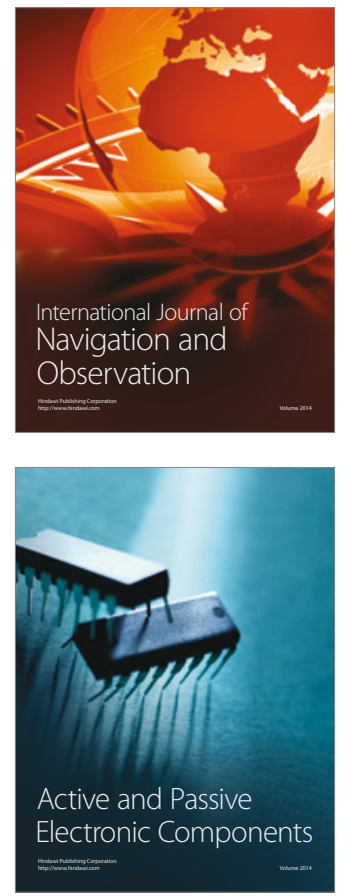
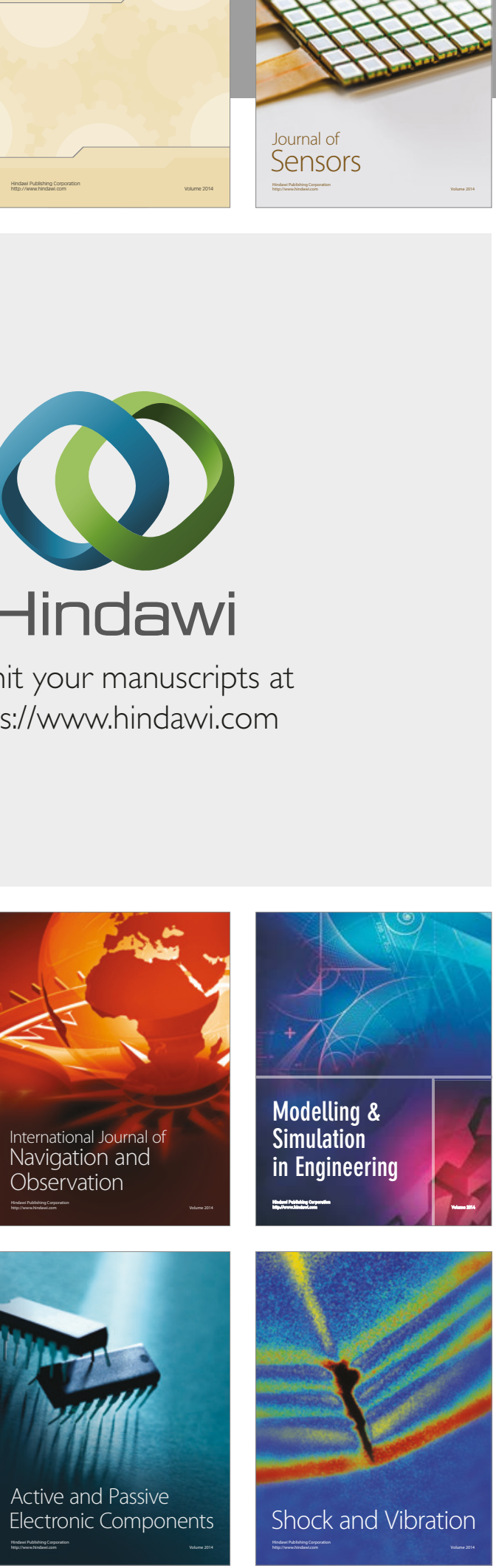
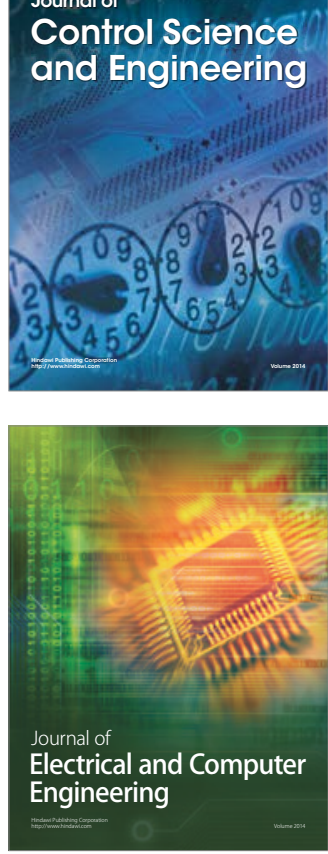

Distributed

Journal of

Control Science

and Engineering
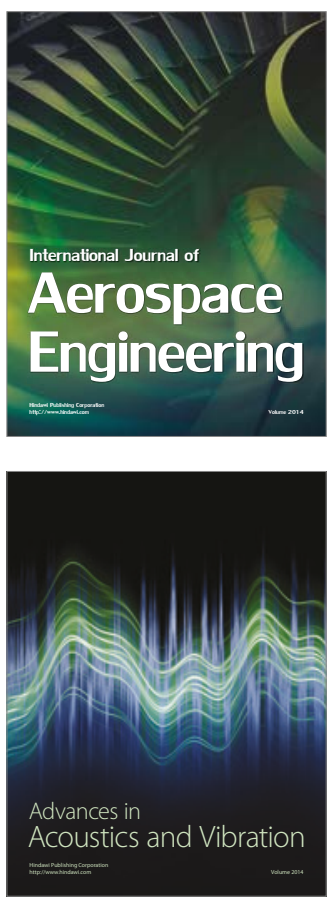

Sensor Networks 